\title{
The influence of the interaction of weight and volume on subjective heaviness
}

\author{
STANLEY J. RULE and DWIGHT W. CURTIS \\ University of Alberta, Edmonton, Alberta, Canada T6G $2 E 9$
}

\begin{abstract}
A model previously proposed for the size-weight illusion (Cross \& Rotkin, 1975) was found unsatisfactory because the postulated volume parameter was not independent of the unit of measurement of weight and the model yielded a paradoxical prediction that the perceived heaviness of small objects should decrease as their weight increased. A new model was proposed based on Stevens and Rubin's (1970) finding that heaviness functions are a family of converging power functions and some of Cross and Rotkin's developments. The model was examined by estimating its parameters from data previously reported by Cross and Rotkin, Stevens and Rubin, and Kolehmainen and Mikkonen (1970). The equations was found to fit each set of data well. Four independent estimates of a volume parameter of the model were in close agreement, $.18, .21, .21$, and .24 , which is consistent with the model.
\end{abstract}

A model intended to describe the joint influence of volume and weight on perceived heaviness is examined in this paper. The model follows from a quantitative description of the size-weight illusion presented by Stevens and Rubin (1970) and an extension of their results by Cross and Rotkin (1975). Stevens and Rubin found that magnitude estimation of the heaviness of objects varying in weight and volume could be described by a family of converging power functions. Each power function described the relation between perceived heaviness and weight when volume was held constant. The exponents and coefficients of the power functions making up the family, varied as a function of volume, such that

$$
\mathbf{Y}=\alpha_{i} \mathbf{W}^{\beta_{\mathrm{i}}}
$$

where $Y$ denotes magnitude estimation of heaviness, $\mathrm{W}$ is physical weight, and parameters $\alpha_{i}$ and $\beta_{i}$ depend on the volume of the ith object. The set of power functions implied by Equation 1 appeared to converge to a point $\left(\mathrm{W}_{\mathrm{c}}, \mathrm{H}_{\mathrm{c}}\right)$, which Stevens and Rubin suggested might represent the response, $\mathrm{H}_{\mathrm{c}}$, corresponding to approximately the heaviest weight, $W_{c}$, the subject might reasonably expect to be able to lift in the experimental situation.

Stevens and Rubin showed that when a set of power functions converge to a point other than the origin, the exponents are a logarithmic function of the coefficients, and Cross and Rotkin showed that the coordinates of the convergence point are the parameters of the function. An expression for this relation is provided by

This research was supported by Grants A0151 and A9582 from the National Research Council of Canada.

$$
\beta_{\mathrm{i}}=-\left(\log \mathrm{W}_{\mathrm{c}}\right)^{-1} \log \alpha_{\mathrm{i}}+\log \mathrm{H}_{\mathrm{c}} / \log \mathrm{W}_{\mathrm{c}} .
$$

An important consequence of Equation 2 is that an expression for the set of power functions can be obtained which contains only one of the parameters, $\alpha_{i}$ or $\beta_{i}$, that is a function of volume in Equation 1 . Either the right side of Equation 2 can be substituted for $\beta_{\mathrm{i}}$ so that only $\alpha_{\mathrm{i}}$ appears in the expression or Equation 2 can be solved for $\alpha_{\mathrm{i}}$ to yield an expression in which only $\beta_{\mathrm{i}}$ appears. The two alternative expressions are

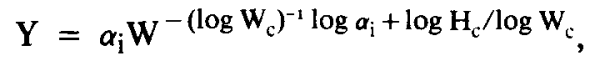

and

$$
\mathrm{Y}=\mathrm{H}_{\mathrm{c}}\left(\frac{\mathrm{W}}{\mathrm{W}_{\mathrm{c}}}\right)^{\beta_{\mathrm{i}}}
$$

Either Equation 3 or Equation 4 can provide a complete mathematical description of the size-weight illusion if the relation between the subscripted parameter and volume can be specified. Because of the ubquitousness of power functions in psychological relations, it seems reasonable to examine the possibility that the relation between one of the subscripted parameters and physical volume is a power function. Cross and Rotkin considered the possibility that the coefficient $\alpha$ is a power function of volume. The present analysis examined the alternative that the exponent $\beta$ is a power function of volume. Our preference for the latter can be justified on theoretical grounds presented below.

\section{Cross and Rotkin's Model}

Cross and Rotkin assumed that $\alpha$ was related to 
volume, $\mathrm{V}$, by a power function with a negative exponent, such that

$$
\alpha=\mathrm{dV}^{-\mathrm{r}} \text {. }
$$

When the right side of Equation 5 was substituted for $a$, Equation 3 was found to provide a very close approximation both to their data and to the data reported by Stevens and Rubin. However, the Cross and Rotkin model may be objected to on two theoretical grounds. First, it can be shown that the value of the exponent for volume, $r$, is dependent on the choice of unit of measurement for weight. Second, the model yields a prediction that when the objects are very small perceived magnitude should decrease as weight increases.

A demonstration that the value of the exponent of the function for volume depends on the unit of measurement for weight is provided by our reanalysis of Cross and Rotkin's data employing pounds rather than grams as the measure of weight. The estimate of $r$ decreased from .98 , obtained in their original analysis, to .36 . An expression can be derived from the Cross and Rotkin model which describes the relative values of two volume exponents $r$ and $r$ ' as a consequence of different weight measures, $W$ and $\mathbf{W}^{\prime}$. Let $\mathbf{W}^{\prime}$ denote a weight measure such that $\mathrm{W}^{\prime}=\mathrm{kW}$ and $\mathrm{W}^{\prime}{ }_{\mathrm{c}}=\mathrm{kW}_{\mathrm{c}}$. The expression is

$$
r / r^{\prime}=\log W_{c} / \log W^{\prime}{ }_{c} .
$$

According to Equation 6, if the Cross and Rotkin model were correct, any estimate of the exponent for volume would be specific to the measure of weight employed in the data analysis. The equation may also explain why the estimate of $r$ from Cross and Rotkin's data (.98) was greater than the estimate from Stevens and Rubin's data (.65). The primary difference between the two studies was that Cross and Rotkin's subjects lifted the objects with both hands, while only one hand was used by Stevens and Rubin's subjects. Cross and Rotkin reasoned that, if $W_{c}$ represented the maximum expected weight that a subject could lift, using two hands should increase its value, a prediction that was confirmed by their data. It can be seen from Equation 6 that an increase in $W_{c}$, because the maximum expected weight was increased due to lifting with both hands, would yield an increase in $r$.

The paradoxical prediction that perceived heaviness should decrease as weight increases when objects are small, follows from an expression derived from the Cross and Rotkin model:

$$
Y=H_{c}\left(\frac{W}{W_{c}}\right)^{\left(r \log V-\log d+\log H_{c}\right) / \log W_{c}}
$$

Consider a small object with volume $\mathrm{V}_{\mathrm{s}}$ such that

$$
\log V_{s} \leqslant\left(\log d-\log H_{c}\right) / r .
$$

According to Equation 7, an object with volume $\mathrm{V}_{\mathrm{s}}$ yields a nonpositive exponent for weight. When the equality in Equation 8 holds, the exponent is zero and the predicted response is the constant $\mathrm{H}_{\mathrm{c}}$ for all values of weight. When the inequality in Equation 8 holds, the exponent is negative and the predicted responses are a decreasing function of weight. They are also greater than $\mathrm{H}_{\mathrm{c}}$ for $\mathrm{W}<\mathrm{w}_{\mathrm{c}}$. Consequently, if the model were correct, small objects, for instance lead shot, would seem heavier than the heaviest weight the subject would expect to be able to lift and they would seem to become lighter as their weight increased. These difficulties with the model are sufficiently serious to reject it as a theoretically appropriate description of the size-weight illusion.

\section{The Proposed Model}

The development of the model to be examined in this paper is focused on the relation between the exponent $\beta$ and volume, which is assumed to be a power function. Also, an additional parameter $\beta_{0}$ is included, which is assumed to be the limiting value of the weight exponent as volume approaches zero. That is, $\beta_{0}$ represents the exponent for weight if volume had no influence on perceived heaviness. The expression for the assumed relation between $\beta$ and volume is

$$
\beta=\beta_{0}+k V q \text {. }
$$

Substitution of the right side of Equation 9 for $\beta_{\mathrm{i}}$ in Equation 4 yields the complete expression for the size-weight illusion that is examined in this paper:

$$
Y=H_{c}\left(\frac{W}{W_{c}}\right)^{\beta_{0}+k V^{q}} .
$$

Equation 10 is more readily compared with the Cross-Rotkin model if the latter is written in an alternative form derived from Equation 7:

$$
Y=H_{c}\left(\frac{W}{W_{c}}\right)^{s+t \log V},
$$

where $s=\left(\log H_{c}-\log d\right) / \log W_{c}$, and $t=r /$ $\log W_{c}$. Equations 10 and 11 are related in that Equation 11 is the limit of Equation 10 as the exponent $q$ approaches zero. It should be noted that the constant $s$ in Equation 11 does not have the same interpretation as $\beta_{0}$ in Equation 10. Instead, $s$ depends on the choice of unit for the measure of 
volume. Both Equations 10 and 11 yield predicted judgments of heaviness that have similar trends for stimuli within the range typically investigated. Perceived heaviness is predicted to decrease with increasing volume, and the relative influence of volume on perceived heaviness decreases as weight increases. The expressions differ, however, in that predictions from Equation 10 for stimuli beyond the range usually investigated are always reasonable. The predicted response is positive and less than $\mathrm{H}_{\mathrm{c}}$ for all objects with a finite volume and weighing less than $\mathrm{W}_{\mathrm{c}}$. Furthermore, the volume exponent $\mathrm{q}$ and the limiting weight exponent $\beta_{\mathrm{o}}$ are independent of the unit of measurement of weight, volume, and the subject's response.

We examined Equation 10 as an appropriate description of the size-weight illusion by estimating its parameters from four sets of data. These were data reported by Cross and Rotkin (1975), Stevens and Rubin (1970), and two sets of data from Kolehmainen and Mikkonen (1970). Goodness of fit might be expected regardless of whether or not the proposed model is appropriate. As noted above, the Cross and Rotkin expression fitted the data well, and the trend in data predicted from Equation 10 is similar to the Cross and Rotkin predictions. Instead, primary consideration in the evaluation of the model was given to how closely estimates of the volume exponent $q$ from the four sets of data agreed.

\section{EXPERIMENTS 1 AND 2}

\section{Method}

Cross and Rotkin (1975) and Stevens and Rubin (1970) used similar methods to obtain judgments of heaviness of objects varying in both weight and volume. Stevens and Rubin's objects ranged in weight from 25 to $6,400 \mathrm{~g}$ and in volume from 58 to $2,325 \mathrm{ml}$. Cross and Rotkin presented objects ranging from 51 to $6,168 \mathrm{~g}$ in weight and from 94 to $4,710 \mathrm{ml}$ in volume. Traditional magnitude estimation procedures, with no modulus designated, were employed in both studies. As noted above, the primary difference between the two studies was that Cross and Rotkin's subjects lifted the objects with both hands, while only one hand was used in Stevens and Rubin's study.

\section{Results}

The expression fitted to the data from each study was the logarithm of Equation 10 expressed as

$$
\log Y=\left(\beta_{o}+k V^{q}\right)\left(\log W-\log W_{c}\right)+\log H_{c} .
$$

The numerical method used for fitting the function was the modified Gauss-Newton method described by Hartley (1961). It is an iterative procedure for finding least squares solutions to nonlinear equations. The sum of squares that was minimized was the squared deviations of the logarithms of predicted and observed
Table 1

Least Squares Estimates of Each Parameter in Equation 10 From Stevens and Rubin's Data and Cross and Rotkins' Data

\begin{tabular}{ccc}
\hline Parameter & $\begin{array}{c}\text { Stevens and } \\
\text { Rubin (1970) }\end{array}$ & $\begin{array}{c}\text { Cross and } \\
\text { Rotkin (1975) }\end{array}$ \\
\hline $\mathrm{H}_{\mathrm{c}}$ & 542 & 238 \\
$\mathrm{~W}_{\mathrm{c}}$ & 6774 & 21445 \\
$\beta_{\mathrm{o}}$ & .84 & .50 \\
$\mathrm{k}$ & .07 & .17 \\
$\mathrm{q}$ & .24 & .18 \\
\hline
\end{tabular}

values. Estimates of parameters obtained from fitting the expression to Stevens and Rubin's data and Cross and Rotkin's data are presented in Table 1. The two estimates of the volume exponent $\mathrm{q}$, the parameter of primary interest, are reasonably close, .18 for Cross and Rotkin's data and .24 for Stevens and Rubin's data.

In Figure 1, magnitude estimates are plotted on semilogarithmic coordinates as a function of volume raised to the $\mathrm{q}^{\text {th }}$ power. The lines through the data represent predicted values based on the parameter estimates presented in Table 1. From Equation 12, the $\log$ predicted values for each uniform weight series can be represented by a line with a slope of $k\left(\log W-\log W_{c}\right)$ and an intercept of $\beta_{o}(\log W$ $\left.-\log W_{c}\right)+\log H_{c}$. The model appears to have provided a good approximation to the data. Each of the two least-squares solutions accounted for over $99 \%$ of the variance in $\log Y$.

The relation between weight exponents $\beta$ and volume predicted from Equation 9 was also examined. For each study, the value of $\beta$ for each uniform volume series was estimated with the restriction that the set of power functions pass through the calculated point of intersection. A strategy of restricting the curves to pass through the same point was used by Stevens, Marks, and Simonson (1974) in their investigation of the joint influence of area and irradiance on perceived warmth. This procedure imposes no limitation on the possible relations between $\beta$ and volume, but it yields a more consistent set of exponents than when unrestricted power functions are fitted independently to each set of from four to six points for each uniform volume series. The exponents obtained from this analysis are plotted in Figure 2 against volume raised to the $\mathrm{q}^{\text {th }}$ power. The slope and intercept of the lines through the plots are the values of $k$ and $\beta_{0}$ presented in Table 1 for each study. Inspection of Figure 2 reveals a good fit to the data.

\section{EXPERIMENTS 3 AND 4}

\section{Method}

The third and fourth sets of data analyzed were reported by Kolehmainen and Mikkonen (1970). These data differed from 

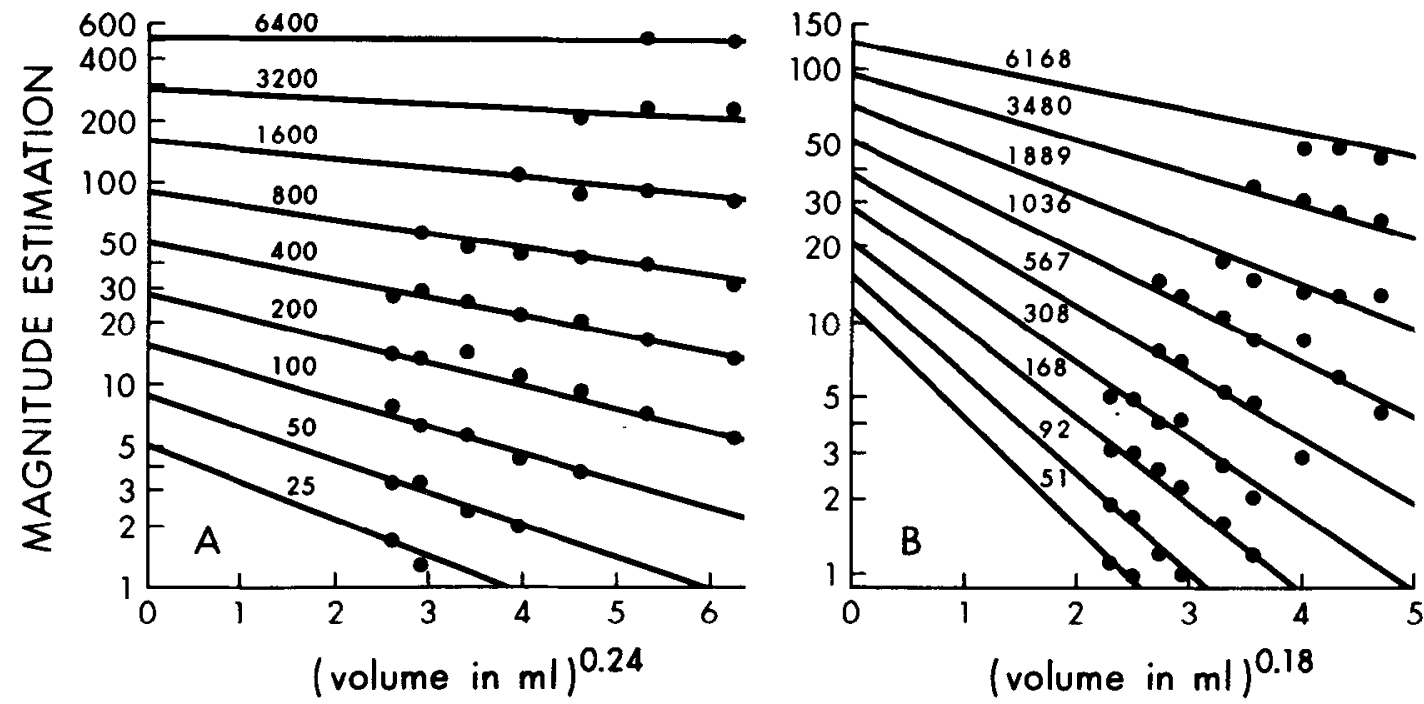

Figure 1. Magnitude estimation for each weight series as a function of volume raised to the power of the estimated value of $q$ for A: A Stevens and Rubin's data (.24) and B: Cross and Rotkin's data (.18). The lines describe least-squares fits of Equation 12 to the data. The parameter is weight in grams.

those just presented in that the objects presented to the subjects varied only in volume. The objects were spheres ranging in volume from 120 to $4,750 \mathrm{ml}$. Each sphere weighed $270 \mathrm{~g}$. One of their sets of data was obtained from ratio estimation of heaviness, while the other set was obtained from magnitude estimation.

\section{Results}

An analysis of the Kolehmainen and Mikkonen data provided an opportunity to examine the fit of the model when only volume varied and to determine whether or not difference in their psychological methods influenced the value of the volume exponent q. Because weight was held constant, an estimate of the point of intersection could not be obtained from the data, and the exponent $q$ is the only meaningful parameter that could be estimated. For objects varying only in volume, the expression for perceived heaviness derived from Equation 10 is the exponential decay function

$$
\mathbf{Y}=\mathrm{ae}^{-\mathrm{bVq}},
$$
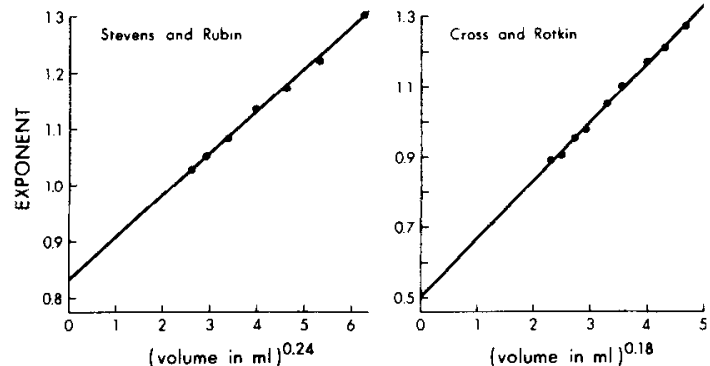

Figure 2. Exponent estimated from magnitude estimation of each uniform volume series as a function of volume raised to the value of $q$ estimated from Stevens and Rubin's data $(.24)$ and Cross and Rotkin's data (.18). The lines are based on parameters presented in Table 1. where

$$
a=H_{c}\left(\frac{W}{W_{c}}\right)^{\beta_{o}}
$$

and

$$
b=-k\left(\log _{e} W-\log _{e} W_{c}\right) \text {. }
$$

Least squares estimates of the parameters of Equation 13 were obtained by the Gauss-Newton method applied to the logarithmic transformation of each side of Equation 13, such that

$$
\log \mathrm{Y}=\log \mathrm{a}-\mathrm{bVq} .
$$

Least squares solutions to Equation 14 for the magnitude estimation data, denoted by $\mathrm{Y}_{\mathrm{ME}}$, and the ratio estimation data, denoted by $\mathrm{Y}_{\mathrm{RE}}$, were provided by

$$
\log \mathrm{Y}_{\mathrm{ME}}=\log 59.1-.36 \mathrm{~V}^{.21},
$$

and

$$
\log \mathrm{Y}_{\mathrm{RE}}=\log 2.3-.14 \mathrm{~V}^{21} .
$$

The agreement in volume exponents suggests that for the two psychophysical procedures investigated, the volume exponent is unaffected by the judgment method. The data from each judgment procedure are plotted on log-linear coordinates in Figure 3 against volume raised to the .21 power. The lines through the plots describe the least squares solutions. In each case the fit was good. 


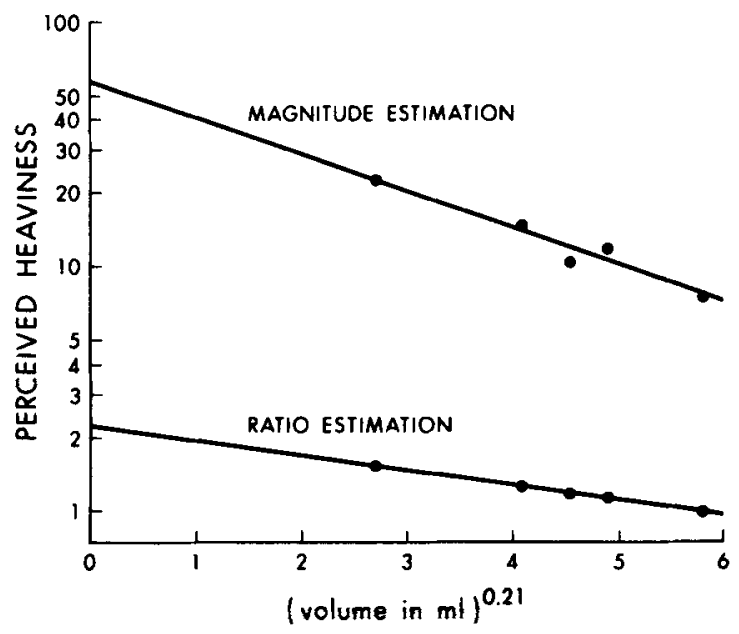

Figure 3. Magnitude estimation and ratio estimation of perceived heaviness reported by Kolehmainen and Mikkonen (1970) as a function of volume raised to the .21 power.

\section{DISCUSSION}

The four estimates of the volume exponent $q$, the parameter of primary interest, were remarkably stable, $.18, .21, .21$, and .24 . This is in marked contrast to the variability of estimates of other parameters. The difference in estimates of $W_{c}$, the maximum weight, from Cross and Rotkin's and Stevens and Rubin's data was to be expected on the basis of whether one or two hands were used to lift the objects. Estimates of $\beta_{0}$ from Cross and Rotkin's and Stevens and Rubin's data also differed considerably, .50 and .84 , respectively. We suggest below that this difference might also be expected on the basis of lifting with one or two hands. In contrast, the volume exponent remains relatively constant with respect to changes in the procedure for lifting the objects and, perhaps, with respect to differences in psychophysical procedures as well.

Equation 10 can also be expressed as

$$
Y=H_{c}\left(\frac{W}{W_{c}}\right)^{\beta_{0}}\left(\frac{W}{W_{c}}\right)^{\beta_{0} k^{\prime} V^{q}},
$$

where $k^{\prime}=k / \beta_{0}$. Expression of the model in this form is useful in examining its implications. Consider first the influence of the object's weight on perceived heaviness in the absence of the object's volume. According to Equation 15, the influence of the object's weight when volume is zero can be expressed as the power function

$$
f(W)=\left(\frac{W}{W_{c}}\right)^{\beta_{0}} .
$$

Equation 16 seems to imply that the weight of an object is evaluated by the subject in reference to an upper weight limit. One might speculate from this result, together with the findings of converging power functions for other modalities (Stevens, 1974), that the neural code for subjective magnitude may be represented as a proportion of the maximum capacity of a sensory channel.

The estimate of $\beta_{0}$ of .50 from Cross and Rotkin's data is much smaller than that of .84 from Stevens and Rubin's data. It should be noted that the lower estimate was not merely a consequence of the model fitted to the data. It is consistent with the generally lower weight exponents that Cross and Rotkin obtained from fitting power functions to heaviness judgments for each uniform volume series. Cross (1973) has shown that a downward bias in the exponent can be produced by sequential effects in the subject's judgments. On the basis of Cross' results, Cross and Rotkin estimated that their weight exponents were reduced by approximately .11 owing to sequential effects. It remains to be shown, however, whether or not Stevens and Rubin's data also were affected in the same way.

Another, perhaps more theoretically interesting, interpretation of the difference in $\beta_{0}$ is suggested by some observations by Teghtsoonian (1971). Teghtsoonian examined an inverse relation, reported by Poulton (1968), between the exponents of various continua and the geometric ranges of the stimulus measures. Teghtsoonian postulated that the relation stemmed from an underlying inverse relation between the exponent and an upper limit of sensitivity. It follows that if the upper limit for a sensory continuum could be increased, the exponent would decrease. Cross and Rotkin, by requiring subjects to lift the objects with both hands, increased the upper limit of heaviness sensitivity, as indicated by $W_{c}$, to approximately three times the upper limit for Stevens and Rubin's subjects. On the basis of Teghtsoonian's theory, the lower weight exponent $\beta_{0}$ obtained from the analysis of Cross and Rotkin's data would be expected as a consequence of the increase in $W_{c}$.

Within the same context, the question arises concerning the possible experimental conditions in which values of $\beta_{\mathrm{i}}$ equal $\beta_{\mathrm{o}}$, and heaviness judgments are obtained that are not influenced by the volume of the objects. Obviously, if the object is not seen by the subject and he does not know its size, the actual physical volume of the object can have no effect on his heaviness judgments. However, it does not follow that the subject's response, when the volume is unseen, is necessarily equivalent to the case in which volume approaches zero. Evidence reported by Ross (1969) on heaviness comparisons of seen and unseen objects indicates that the volume for an observed object can be found such that the seen object yields the same perceived heaviness as an unseen object of the same weight. Her result would seem to imply 
that an unseen object is not equivalent to one with zero volume. An alternative basis for judgments of heaviness not influenced by perceived volume is suggested by some phenomenological observations reported by Koseleff (1957). According to Koseleff, perceived heaviness is experienced as a property of the object and external to the observer. He suggested that, if the subject concentrated on his organic sensations of pressure and strain rather than on the object, the volume of the object would have no influence on his heaviness judgments. Perhaps $\beta_{o}$ is the exponent for judged heaviness when the subject is instructed to attend to his sensory experience rather than to the physical object. On the other hand, $\beta_{0}$ may be a parameter for a theoretical limit, that of volume approaching zero, which is not obtained in practice.

The remaining terms in Equation 15 can be expressed as an interaction component for weight and volume:

$$
h(W, V)=f(W)^{g(V)}
$$

where

$$
g(V)=k^{\prime} V^{q} \text {. }
$$

The nature of the Weight by Volume interaction $h$ can be seen by examining the influence of volume on $h(W, V)$ when weight is held constant. The relation between $h(W, V)$ and $g(V)$ for a constant weight is the exponential decay function

$$
h(W, V)=e^{-b^{\prime} g(V)}
$$

where

$$
b^{\prime}=-\log _{\mathrm{e}} f(W) \text {. }
$$

To illustrate the form of the function expressed as Equation 18, a family of exponential functions have been graphed in Figure 4. Each curve represents the value of the interaction as a function of $g(V)$ for a constant weight. The lower curves, in particular, provide an illustration of a law of diminishing returns for the influence of volume. A constant increment to the value of the volume function $g(V)$ yields a greater change in $h(W, V)$ for lower values of $g(V)$ than for higher ones. Note that this effect becomes less pronounced with successively higher curves. This trend illustrates the nature of the Weight by Volume interaction $h$. The rate of decay in $h(W, V)$ with $g(V)$ is a decreasing function of weight. At the limit when weight equals $W_{c}$, volume has no influence on perceived heaviness, as illustrated by the horizontal line at the top of Figure 4. Perhaps the interaction $h$

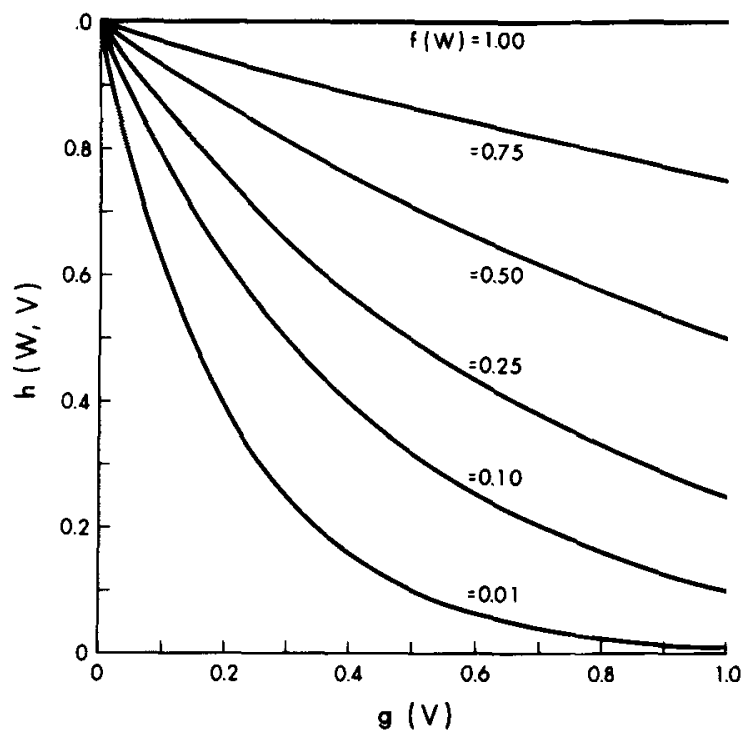

Figure 4. A family of exponential decay functions in which values of the interaction $h$ are graphed as a function of values of the volume function $g$. Each curve represents a different value of the weight function $f$. See text for a specification of the functions.

could be well summarized as a diminishing law of diminishing returns for the joint influence of weight and volume on perceived heaviness.

\section{REFERENCES}

Cross, D. V. Sequential dependencies and regression in psychophysical judgments. Perception \& Psychophysics, 1973, 14, 547-552.

Cross, D. V., \& Rotrin, L. The relation between size and apparent heaviness. Perception \& Psychophysics, 1975, 18, 79-87.

Hartley, H. O. The modified Gauss-Newton method for the fitting of non-linear regression functions by least squares. Technometrics, 1961, 3, 269-280.

KoselefF, P. Studies in the perception of heaviness. I. Acta Psychologica, 1957, 13, 242-252.

Kolehmainen, K., \& Mikronen, V. Apparent weight differences as a source of after-effects. Scandinavian Journal of Psychology, $1970,11,161-166$.

Poulton, E. C. The new psychophysics: Six models for magnitude estimation. Psychological Bulletin, 1968, 69, 1-19.

Ross, H. E. When is a weight not illusory? Quarterly Journal of Experimental Psychology, 1969, 21, 346-355.

Stevens, J. C. Families of converging power functions in psychophysics. In H. R. Moskowitz, B. Scharf, \& J. C. Stevens (Eds.), Sensation and measurement: Papers in honor of S. S. Stevens. Dordrecht, The Netherlands: Reidel, 1974.

Stevens, J. C., Marks, L. E., \& Simonson, D. C. Regional sensitivity and spatial summation in the warmth sense. Physiology and Behavior, 1974, 13, 825-836.

Stevens, J. C., \& Rubin, L. L. Psychophysical scales of apparent heaviness and the size-weight illusion. Perception \& Psychophysics, 1970, 8, 225-230.

Teghtsoonian, R. On the exponents in Stevens' law and the constant in Ekman's law. Psychological Review, 1971, 78, 71-80.

(Received for publication October 15, 1976; revision accepted May 24, 1977.) 